ISAHP 2005, Honolulu, Hawaii, July 8-10, 2005

\title{
AHP for Game Theoretic Decision Making
}

\author{
Masaaki Shinohara \\ Nihon University \\ Izumi-chou, Narashino \\ Chiba 275-8575, Japan \\ M7sinoha@cit.nihon-u.ac.jp
}

\author{
Keikichi Osawa \\ Nihon University \\ Izumi-chou, Narashino \\ Chiba 275-8575, Japan \\ k7oosawa@cit.nihon-u.ac.jp
}

\section{Ken Shinohara}

Institute of Information Systems

Hikarigaoka, Nerima

Tokyo 179-0072, Japan

m7sinoha@cit.nihon-u.ac.jp

Keywords: zero-sum game, payoff matrix, decomposition, strategy, tactics

Summary: Hierarchical decomposition is applied to the payoff matrix of a 2-player zero-sum noncooperative game. Relationship between the strategy vector of the integrated payoff matrix and the strategy vector of each decomposed payoff matrix is studied. For the case of $2 \times 2$ zero-sum noncooperative game with purely mixed strategies, a simple formula which relates the strategy vector of the integrated payoff matrix and the strategy vector of each decomposed payoff matrix is established; the strategy vector of the integrated payoff matrix is obtained as a weighted combination of the strategy vector of each decomposed payoff matrix with the weight considering both the priority weight of each decomposed payoff matrix (or payoff matrix for each criterion) and the magnitude of each decomposed payoff matrix. The higher layer integrated game corresponds to the strategic decision making and the lower layer decomposed games correspond to the tactical decision making.

\section{Introduction}

In the framework of game theoretic decision making, there are two or more players competing each other so as to optimize each of their own goals. Likely outcomes when players select from various courses of strategies are called payoffs. When the payoffs are expressed in the form of matrix, they are called payoff matrix. Each element of a payoff matrix for a player is the outcome of the game for the player when the player and the rest of players take some specific combination of strategies. We apply the hierarchical decomposition to the payoff matrix of a game, or namely, we consider the situation where the payoff matrix consists of several submatrixes with different criterion(Fig.1)

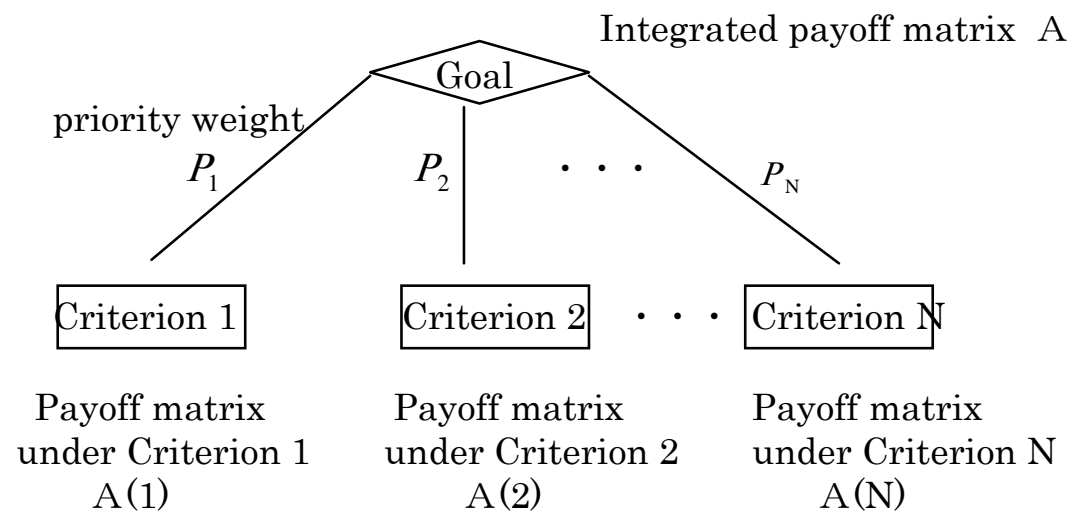

Fig.1 Hierarchical decomposition of payoff matrix

For the case of $2 \times 2$ zero-sum noncooperative game, we will investigate the relationship between the minimax-maximin equilibrium solution of an integrated 
payoff matrix and the equilibrium solution of each decomposed payoff matrix.

In Chapter 2, we will show an example where the strategy vector of an integrated payoff matrix cannot be obtained by simply weighting each solution vector by its priority weight and combining them. In Chapter 3 , we will prove a theorem stating that the strategy vector of an integrated payoff matrix can be obtained by the weighted sum of each solution vector considering not only the priority weight but also the characteristic weight of each decomposed payoff matrix. In Chapter 4, the validity of presented theorem is examined by three $2 \times 2$ zero-sum game examples. In Chapter 5 , future research problems are discussed from the viewpoint of game-theoretic AHP.

\section{Coin matching game}

Consider the following $2 \times 2$ zero-sum game of matching coins. There are two players, player I and player II. Player I has two strategies to show heads $\mathrm{H}$ or tails T, and player II can likewise select $\mathrm{H}$ or T. If the two players' coins match with either two heads or else two tails, then player I wins $\$_{4} \frac{2}{3}$ or $\$_{6} \frac{1}{3}$, respectively, from player II . If the coins do not match, player I wins less moneys from player II as shown by the payoff matrix A of Eq.(1). Because of coin matching game, player I gets more moneys from player II when the coins do match, and the payoff matrix A shows moneys player I gets from player II for the four cases.

$$
\begin{gathered}
\mathrm{H} \\
\mathrm{A}=\mathrm{I} \\
\mathrm{H} \\
\mathrm{T}
\end{gathered}\left(\begin{array}{cc}
\frac{14}{3} & \frac{5}{3} \\
\frac{4}{3} & \frac{19}{3}
\end{array}\right)
$$

Actually this payoff matrix is determined from the viewpoint of two factors (or two criteria). One is stability factor and the other is speculation (or gambling) factor. From the viewpoint of stability, the amounts of moneys player I gets from player II when the coins do match should be not so big from those when they do not match. So the payoff matrix A(1) under the stability criterion(Criterion 1) would be given by Eq.(2).

$$
A(1)=\left(\begin{array}{ll}
4 & 1 \\
2 & 3
\end{array}\right)
$$

From the viewpoint of speculation, the amounts of moneys player I gets from player II when the coins do match should be far bigger than those when they do not match. So the payoff matrix A(2) under the speculation criterion(Criterion 2) would be given by Eq.(3).

$$
A(2)=\left(\begin{array}{ll}
5 & 2 \\
1 & 8
\end{array}\right)
$$

If the importance ratio of Criterion 1 to Criterion 2 is $1: 2$, then the integrated payoff matrix A of Eq.(1) is obtained by Eq.(4), where $P_{1}=1 / 3$ and $P_{2}=2 / 3$. 


$$
\mathrm{A}=P_{1} \mathrm{~A}(1)+P_{2} \mathrm{~A}(2)
$$

Equilibrium solutions for the integrated payoff matrix A, or maximin strategy vector $x$ for player I and minimax strategy vector $y$ for player II, are given by Eq.(5) and Eq.(6)

$$
\begin{aligned}
& x=\left(\begin{array}{l}
5 / 8 \\
3 / 8
\end{array}\right) \\
& y=\left(\begin{array}{l}
7 / 12 \\
5 / 12
\end{array}\right)
\end{aligned}
$$

Equilibrium solutions for the payoff matrix A(1) are given by Eq.(7) and Eq.(8)

$$
\begin{aligned}
& x(1)=\left(\begin{array}{l}
1 / 4 \\
3 / 4
\end{array}\right) \\
& y(1)=\left(\begin{array}{l}
1 / 2 \\
1 / 2
\end{array}\right)
\end{aligned}
$$

Equilibrium solutions for the payoff matrix A(2) are given by Eq.(9) and Eq.(10).

$$
\begin{aligned}
& x(2)=\left(\begin{array}{l}
0.7 \\
0.3
\end{array}\right) \\
& y(2)=\left(\begin{array}{l}
0.6 \\
0.4
\end{array}\right)
\end{aligned}
$$

Among the equilibrium solutions of the three payoff matrixes, we hope that some relationship should hold, such as Eq.(11) and Eq.(12)

$$
\begin{aligned}
& x=P_{1} x(1)+P_{2} x(2) ? \\
& y=P_{1} y(1)+P_{2} y(2) ?
\end{aligned}
$$

But Eq.(11) and Eq.(12) do not hold. That is, the strategy vector of an integrated payoff matrix cannot be obtained in general by simply weighting the strategy vector of each decomposed payoff matrix by its priority weight and combining them.

\section{AHP Theorem for game theoretic decision making}

We will present a theorem which relates equilibrium strategy vectors of an integrated payoff matrix to equilibrium strategy vectors of decomposed payoff matrixes.

[Theorem]

Consider a 2-player $2 \times 2$ zero-sum game with payoff matrix $\mathrm{A}=\left(\begin{array}{ll}a_{11} & a_{12} \\ a_{21} & a_{22}\end{array}\right)$ for maximizer player I, and let $x$ and $y$ be equilibrium strategy vectors for player I and player II, respectively. Let $\mathrm{A}(\mathrm{k})=\left(\begin{array}{ll}a_{11}(k) & a_{12}(k) \\ a_{21}(k) & a_{22}(k)\end{array}\right)$ be the $k$ th decomposed payoff 
matrix under the $k$ th criterion $(k=1, \ldots, \mathrm{N})$ and $x(k)$ and $y(k)$ be its equilibrium strategy vectors for player I and player II, respectively. Then, following relationship holds, if $0<x<1,0<y<1,0<x(k)<1$ and $0<y(k)<1$.

$$
\begin{aligned}
& x=\sum \frac{p_{k} \Delta_{k}}{\Delta} x(k) \\
& y=\sum \frac{p_{k} \Delta_{k}}{\Delta} y(k)
\end{aligned}
$$

Here, $p_{k}$ is the priority weight for the $k$ th criterion, and $\Delta_{k}$ and $\Delta$ are given by Eq.(15) and Eq.(16).

$$
\begin{aligned}
& \Delta_{k}=a_{11}(k)+a_{22}(k)-a_{12}(k)-a_{21}(k) \quad k=1, \ldots, \mathrm{N} \\
& \Delta=\sum p_{k} \Delta_{k}
\end{aligned}
$$

[Proof]

Under the condition of $0<x<1,0<y<1,0<x(k)<1$ and $0<y(k)<1, \quad x, y$, $x(k)$ and $y(k)$ are explicity given as below.

$$
\begin{aligned}
& x=\left(\begin{array}{c}
\frac{a_{22}-a_{21}}{a_{22}+a_{11}-a_{12}-a_{21}} \\
\frac{a_{11}-a_{12}}{a_{22}+a_{11}-a_{12}-a_{21}}
\end{array}\right) \\
& y=\left(\begin{array}{c}
\frac{a_{22}-a_{12}}{a_{22}+a_{11}-a_{12}-a_{21}} \\
\frac{a_{11}-a_{21}}{a_{22}+a_{11}-a_{12}-a_{21}}
\end{array}\right) \\
& x(k)=\left(\begin{array}{c}
\frac{a_{22}(k)-a_{21}(k)}{a_{22}(k)+a_{11}(k)-a_{12}(k)-a_{21}(k)} \\
\frac{a_{11}(k)-a_{12}(k)}{a_{22}(k)+a_{11}(k)-a_{12}(k)-a_{21}(k)}
\end{array}\right) \\
& y(k)=\left(\begin{array}{c}
\frac{a_{22}(k)-a_{12}(k)}{a_{22}(k)+a_{11}(k)-a_{12}(k)-a_{21}(k)} \\
\frac{a_{11}(k)-a_{21}(k)}{a_{22}(k)+a_{11}(k)-a_{12}(k)-a_{21}(k)}
\end{array}\right) \quad k=1, \ldots, \mathrm{N} \\
& k=1, \ldots, \mathrm{N}
\end{aligned}
$$

Substituting $a_{i j}=\sum a_{i j}(k)$, Eq.(15), Eq.(16), Eq.(19) and Eq.(20) into the right-hand sides of Eq.(13) and Eq.(14), the relationship (13) and (14) is directly proved.(Q.E.D.)

This AHP theorem for game theoretic decision making holds under the condition of $0<x<1,0<y<1,0<x(k)<1$ and $0<y(k)<1$, where any element of strategy vectors is not allowed to take neither 0 nor 1 . So, this condition can be called "purely mixed strategy condition". This AHP theorem is expected to hold even not under purely 
mixed strategy condition, which will be examined by Example 3 in the next chapter.

The AHP theorem states that strategy vectors of an integrated game can be expressed by weighted average of strategy vectors of each decomposed game with weight $p_{k} \Delta_{k}$, where $p_{k}$ is the priority weight for the $k$ th criterion and $\Delta_{k}$ can be interpreted as indicating the magnitude of each decomposed game. Also note that $\Delta$ (defined by Eq.(16)) can be interpreted as the magnitude of the integrated game.

\section{Examples}

The validity of the AHP theorem in Chapter 3 will be examined by there $2 \times 2$ zero-sum game examples.

\subsection{Example 1; coin matching game}

The AHP theorem is applied to the coin matching game in Chapter 2, where the purely mixed strategy condition is satisfied.

Instead of Eq.(11) and Eq.(12), the AHP theorem insists that Eq.(21) and Eq.(22) hold.

$$
\begin{gathered}
x=\frac{p_{1} \Delta_{1}}{\Delta} x(1)+\frac{p_{2} \Delta_{2}}{\Delta} x(2) \\
y=\frac{p_{2} \Delta_{2}}{\Delta} y(1)+\frac{p_{2} \Delta_{2}}{\Delta} y(2)
\end{gathered}
$$

Substituting $p_{1}=1 / 3, p_{2}=2 / 3, \Delta_{1}=4, \Delta_{2}=10, \Delta=8$, Eqs.(7), (8), (9) and (10) into right-hand sides of Eq.(21) and (22), $x$ and $y$ are calculated, which exactly coincide with those in Eq.(5) and Eq.(6).

\subsection{Example 2; TV program broadcasting game}

Two TV stations are wondering which TV programs to broadcast, "drama" or "quiz", on the prime time. The integrated payoff matrix A for player I is given by Eq.(23), which is made of two payoff matrixes, A(1) of Eq.(24) and A(2) of Eq.(25).

$$
\begin{aligned}
& \mathrm{A}=\underset{\text { quiz }}{\operatorname{drama}}\left(\begin{array}{cc}
1.4 & -0.4 \\
0.2 & 0.6
\end{array}\right) \\
& \mathrm{A}(1)=\left(\begin{array}{cc}
2 & -1 \\
0 & 1
\end{array}\right) \\
& \mathrm{A}(2)=\left(\begin{array}{cc}
-1 & 2 \\
1 & -1
\end{array}\right)
\end{aligned}
$$

$\mathrm{A}(1)$ is the payoff matrix from the viewpoint of attractiveness to TV viewers and $\mathrm{A}(2)$ is the payoff matrix from the viewpoint of commercial effect. The integrated payoff matrix is obtained as a weighted sum of these two payoff matrixes, with priority weight $p_{1}$ on Criterion 1 (attractiveness) being 0.8 and priority weight $p_{2}$ on Criterion 2 (commercial effect) being 0.2 .

$$
\begin{aligned}
& \mathrm{A}=p_{1} \mathrm{~A}(1)+p_{2} \mathrm{~A}(2) \\
& p_{1}=0.8, \quad p_{2}=0.2
\end{aligned}
$$


The equilibrium solutions for the three payoff matrixes are given as below:

$$
\begin{aligned}
& x=\left(\begin{array}{c}
\frac{2}{11} \\
\frac{9}{11}
\end{array}\right) \\
& y=\left(\begin{array}{l}
\frac{5}{11} \\
\frac{6}{11}
\end{array}\right) \\
& x(1)=\left(\begin{array}{l}
0.25 \\
0.75
\end{array}\right) \\
& y(1)=\left(\begin{array}{l}
0.5 \\
0.5
\end{array}\right) \\
& x(2)=\left(\begin{array}{l}
0.4 \\
0.6
\end{array}\right) \\
& y(2)=\left(\begin{array}{l}
0.6 \\
0.4
\end{array}\right)
\end{aligned}
$$

Since the magnitude of the game under Criterion 2 is negative $\left(\Delta_{2}=-5\right), x(y)$ is not a convex combination of $x(1)$ and $x(2)(y(1)$ and $y(2))$, but following equations of AHP Theorem do hold.

$$
\begin{aligned}
& x=\frac{p_{1} \Delta_{1}}{\Delta} x(1)+\frac{p_{2} \Delta_{2}}{\Delta} x(2) \\
& y=\frac{p_{2} \Delta_{2}}{\Delta} y(1)+\frac{p_{2} \Delta_{2}}{\Delta} y(2)
\end{aligned}
$$

Here, $\frac{p_{1} \Delta_{1}}{\Delta}=\frac{16}{11}(>0)$ and $\frac{p_{2} \Delta_{2}}{\Delta}=-\frac{5}{11}(<0)$.

\subsection{Example 3; Offense-defense fighting game}

Two forces are fighting each other and wondering which strategy to take, "offense" or "defense". The integrated payoff matrix A for player I is given by Eq.(35), which is made of two payoff matrixes, A(1) of Eq.(36) and A(2) of Eq.(37).

$$
\begin{aligned}
& \mathrm{A}=\begin{array}{l}
\text { offense } \\
\text { defense }
\end{array}\left(\begin{array}{ll}
0.4 & 1.4 \\
0.2 & 2.7
\end{array}\right) \\
& \mathrm{A}(1)=\left(\begin{array}{cc}
1 & 2 \\
-1 & 3
\end{array}\right) \\
& \mathrm{A}(2)=\left(\begin{array}{cc}
-1 & 0 \\
3 & 2
\end{array}\right)
\end{aligned}
$$

$\mathrm{A}(1)$ is the payoff matrix from the viewpoint of weapon ability and A(2) is the payoff matrix from the viewpoint of social justice. The integrated payoff matrix is obtained as a 
weighted sum of these two payoff matrixes, with priority weight $p_{1}$ on Criterion 1 (weapon) being 0.7 and priority weight $p_{2}$ on Criterion 2 (social justice) being 0.3 .

$$
\begin{aligned}
& \mathrm{A}=p_{1} \mathrm{~A}(1)+p_{2} \mathrm{~A}(2) \\
& p_{1}=0.7, \quad p_{2}=0.3
\end{aligned}
$$

The equilibrium solutions for the three payoff matrixes are given as below:

$$
\begin{aligned}
& x=\left(\begin{array}{l}
1 \\
0
\end{array}\right) \\
& y=\left(\begin{array}{l}
1 \\
0
\end{array}\right) \\
& x(1)=\left(\begin{array}{l}
1 \\
0
\end{array}\right) \\
& y(1)=\left(\begin{array}{l}
1 \\
0
\end{array}\right) \\
& x(2)=\left(\begin{array}{l}
0 \\
1
\end{array}\right) \\
& y(2)=\left(\begin{array}{l}
0 \\
1
\end{array}\right)
\end{aligned}
$$

Since all the equilibrium solutions are pure strategies and the purely mixed strategy condition is not satisfied, AHP Theorem does not hold in its original form as shown below.

$$
\begin{aligned}
& \frac{p_{1} \Delta_{1}}{\Delta} x(1)+\frac{p_{2} \Delta_{2}}{\Delta} x(2)=\left(\begin{array}{c}
\frac{7}{5} \\
-\frac{2}{5}
\end{array}\right) \neq x \\
& \frac{p_{2} \Delta_{2}}{\Delta} y(1)+\frac{p_{2} \Delta_{2}}{\Delta} y(2)=\left(\begin{array}{c}
\frac{7}{5} \\
-\frac{2}{5}
\end{array}\right) \neq y
\end{aligned}
$$

But if $\frac{7}{5}$ is truncated to 1.0 and $-\frac{2}{5}$ is truncated to 0 , then the equality holds both in (45) and (46). Even when the AHP Theorem in its original form does not hold because of the violation of the purely mixed strategy condition, there remains a possibility that the AHP Theorem holds in a variant form, as shown by this Example 3.

\section{Discussions}

\subsection{Strategic decision making and tactical decision making}

When a game is decomposed in a hierarchical manner as in AHP, the original game 
corresponds to strategic goal decision making, and each of decomposed game corresponds to tactical decision making. So the AHP Theorem shows how the strategic decision making is related to each of tactical decision makings

\subsection{Normalizing games}

The AHP Theorem in Chapter 3 states that the integrated strategy $x$ is obtained by weighted average of each substrategy (or tactics) $x(i)$ with weight $p_{i} \Delta_{i}$, priority weight of each criterion multiplied by its game magnitude. That is, in order to form the integrated strategy from substrategies, we have to take the structure of payoff matrix under each criterion into consideration.

If you want a simpler form of AHP Theorem for game theoretic decision making, you need to normalize each payoff matrix $\mathrm{A}(k)$ by its magnitude $a_{11}(k)+a_{22}(k)-a_{12}(k)-a_{21}(k)$.

\subsection{Game theoretic AHP in general}

The game theoretic AHP for the case of 2-player zero-sum noncooperative $2 \times 2$ matrix game has been discussed in this paper. What would the generalized version of game theoretic AHP be? For the case of 2-player $m \times n$ payoff matrix, it would be like; player I (decision maker I) has $m$ strategies ( $m$ alternatives) to choose from and player II (decision maker II) has $n$ strategies $(n$ alternatives) to choose from. This situation is described by an $m \times n$ payoff matrix $A_{1}$ for player I and an $m \times n$ payoff matrix $\mathrm{A}_{2}$ for player II.

When $A_{1}+A_{2}=0$, it is a zero-sum game and when $A_{1}+A_{2} \neq 0$, it is a nonzero-sum game. Each payoff matrix $A_{1}$ or $A_{2}$ is estimated in consideration of $\mathrm{N}$ criteria.

$$
\begin{aligned}
& \mathrm{A}_{1}=p_{1} \mathrm{~A}_{1}(1)+p_{2} \mathrm{~A}_{1}(2)+\ldots+p_{\mathrm{N}} \mathrm{A}_{1}(\mathrm{~N}) \\
& \mathrm{A}_{2}=q_{1} \mathrm{~A}_{2}(1)+q_{2} \mathrm{~A}_{2}(2)+\ldots+q_{\mathrm{N}} \mathrm{A}_{2}(\mathrm{~N})
\end{aligned}
$$

Here, $p_{k}\left(q_{k}\right)$ is the priority weight on Criterion $k$ for player I ( II ), and $\mathrm{A}_{1}(k)\left(\mathrm{A}_{2}(k)\right)$ is $m \times n$ payoff matrix under Criterion $k$ for player I (II).

One of the problems for the 2-player matrix game would be to investigate the relationship between equilibrium solutions for $A_{1}$ and $A_{2}$ and equilibrium solutions for each of $\mathrm{A}_{1}(k)$ and $\mathrm{A}_{2}(k)$.

\subsection{Game theoretic AHP in application}

If all the payoff matrixes under the criteria are known, the effectiveness of the AHP Theorem for XXX decreases, because the AHP Theorem only reconfirms the calculated result of taken strategies for the integrated payoff matrix. The AHP Theorem becomes effective when the payoff matrixes are not known, but the strategies under decomposed criteria and the importance of each criterion are known. This is a case where total judgment (or strategic decision) is made at company headquarter who knows only each local judgment (or tactical decision) made at each company division. 


\section{Conclusion}

Decomposing payoff matrix along hierarchy, AHP Theorem for game theoretic decision making has been established for the case of 2-player zero-sum noncooperative $2 \times 2$ matrix game (Chapter 3).

Generalization and extention of presented AHP Theorem beyond the case of 2-player zero-sum noncooperative $2 \times 2$ matrix game is future research issues. Game theoretic ANP is also a future research theme, where individual subgames are related in a network manner.

\section{References}

[1] T.L.Saaty :The Analytic Hierarchy Process(Mc-Graw Hill)(1980)

[2] T.L.Saaty :The Analytic Network Process(RWS-Publication)(1996) 